Research

\title{
Competency of Anopheles stephensi mysorensis strain for Plasmodium vivax and the role of inhibitory carbohydrates to block its sporogonic cycle
}

\author{
Hamid R Basseri*1, Soghra Doosti ${ }^{1}$, Kamran Akbarzadeh ${ }^{1}$, \\ Mehdi Nateghpour ${ }^{1}$, Miranda MA Whitten ${ }^{2}$ and Hossein Ladoni ${ }^{1}$
}

Address: ${ }^{1}$ Department of Medical Entomology, School of Public Health, Tehran University of Medical Science, Iran and ${ }^{2}$ School of the Environment and Society, Swansea University, Singleton Park, Swansea, SA2 8PP, UK

Email: Hamid R Basseri* - Basserih@sina.tums.ac.ir; Soghra Doosti - DoostiS@yahoo.com; Kamran Akbarzadeh - akbarzadehk@yahoo.com; Mehdi Nateghpour - NategpourM@sina.tums.ac.ir; Miranda MA Whitten - M.M.A.Whitten@swansea.ac.uk ;

Hossein Ladoni - ladonih@yahoo.com

* Corresponding author

Published: 15 July 2008

Malaria Journal 2008, 7:131 doi:10.1|186/1475-2875-7-131
Received: 17 November 2007

Accepted: I5 July 2008

This article is available from: http://www.malariajournal.com/content/7////3I

(c) 2008 Basseri et al; licensee BioMed Central Ltd.

This is an Open Access article distributed under the terms of the Creative Commons Attribution License (http://creativecommons.org/licenses/by/2.0), which permits unrestricted use, distribution, and reproduction in any medium, provided the original work is properly cited.

\begin{abstract}
Background: Despite the abundance of studies conducted on the role of mosquitoes in malaria transmission, the biology and interaction of Plasmodium with its insect host still holds many mysteries. This paper provides the first study to follow the sporogonic cycle of Plasmodium vivax in a wild insecticide-resistant mysorensis strain of Anopheles stephensi, a major vector of vivax malaria in south-eastern Iran. The study subsequently demonstrates that host-parasite sugar binding interactions are critical to the development of this parasite in the salivary glands of its mosquito host. The identity of the receptors or sugars involved was revealed by a receptor "pre-saturation" strategy in which sugars fed to the mosquitoes inhibited normal host-parasite interactions.

Methods: Anopheles stephensi mysorensis mosquitoes were artificially infected with $P$. vivax by feeding on the blood of gametocytaemic volunteers reporting to local malaria clinics in the Sistan-Baluchistan province of southeastern Iran. In order to determine the inhibitory effect of carbohydrates on sporogonic development, vector mosquitoes were allowed to ingest blood meals containing both gametocytes and added carbohydrates. The carbohydrates tested were GlcNAc, GalNAc, arabinose, fucose, mannose, lactose, glucose and galactose. Sporogonic development was assessed by survival of the parasite at both the oocyst and sporozoite stages.

Results: Oocyst development was observed among nearly $6 \%$ of the fed control mosquitoes but the overall number of mosquitoes exhibiting sporozoite invasion of the salivary glands was $47.5 \%$ lower than the number supporting oocysts in their midgut. Of the tested carbohydrates, only arabinose and fucose slightly perturbed the development of $P$. vivax oocysts at the basal side of the mosquito midgut, and the remaining sugars caused no reductions in oocyst development. Strikingly however, sporozoites were completely absent from the salivary glands of mosquitoes treated with mannose, GalNAc, and lactose.

Conclusion: The study indicates that An. stephensi in southern Iran has the potential to survive long enough to be re-infected and transmit vivax malaria several times, based on the average adult female longevity (about 30 days) and its gonotrophic cycle ( $2-3$ days) during the malaria transmission season. Certain sugar binding interactions are important for the development of $P$. vivax sporozoites, and this information may be instrumental for the development of transmission blocking strategies.
\end{abstract}




\section{Background}

Malaria remains the most devastating vector-borne disease despite a century of concerted international efforts to control it. Most antimalarial strategies have primarily targeted the infection in humans and the control of mosquitoes with insecticides. These approaches have proven unsuccessful, in part because parasites have developed resistance to antimalarial drugs, and mosquitoes have become resistant to insecticides. The malaria parasite Plasmodium takes many forms during its complex life cycle in the vertebrate host and invertebrate vector [1]. Our understanding of the basic biology of vector-parasite interactions during the transit of the parasite through the mosquito is still limited [2]. Upon ingestion, Plasmodium gametocytes differentiate into gametes within the midgut lumen, fertilization takes place and the zygote develops into an ookinete, which penetrates the midgut epithelium and forms oocysts on the basal side. When the oocysts mature and subsequently rupture, sporozoites are released into the haemolymph and invade the salivary glands, to be injected into a new host when the infected mosquito feeds again. Diverse mosquito species differ in their ability to transmit different Plasmodium parasites, and stage-to-stage specific losses seem to depend on the vector and the parasite species [3]. A certain mosquito species always shows different permissiveness to different Plasmodium species. For instance, Plasmodium berghei will develop at least 50 oocysts in Anopheles gambiae, while Plasmodium falciparum rarely exceeds three to four oocysts per midgut in the field and even in laboratory infections [4].

There are several possible new approaches to interrupt the malaria transmission cycle in the mosquito, including mosquito vaccines [2], transmission-blocking vaccines [3] or transmission-blocking antimalarial drugs [5,6]. Understanding the life cycle of medically-important Plasmodium species/strain combinations is crucial for the development of vaccines, and the design of an effective transmission-blocking strategy depends on a full molecular understanding of the sporogonic development of the parasite in the mosquito [2]. During sporogony, a parasite's transition from one developmental stage to another is confined to specific tissue compartments within the mosquito. Each compartment contains specific factors that interact with the parasite and influence its development. Potentially the role of carbohydrate receptors represents one of the most important keys to understanding vector/ parasite interactions [7]. A variety of sugar linkages are present on the surface of the mosquito midgut epithelium, some of which partially block attachment of malaria ookinetes to the midgut surface in vitro [8]. Thus, the mosquito midgut epithelium, similar to the lining of mammalian intestines, is extensively covered with surface carbohydrates that may play a role in pathogen attachment.

Recently evidence for a "protein-carbohydrate recognition strategy" for blocking vector host-pathogen interactions has emerged. Through a combination of conventional and novel approaches, oligosaccharide mimics or antiglycan antibodies are being developed that could help to reduce disease transmission [9]. For example, antiglycan antibodies that are taken up by a mosquito along with a parasite infective blood meal, can mask parasite glycan receptors that are critical for attachment to the midgut [10]. In addition, glycoproteins are potential transmission-blocking vaccine candidates for insect vector-borne diseases and further reinforce the importance of understanding the effects of carbohydrate epitopes in development of vaccines [11]. Anti-carbohydrate antibodies successfully block Plasmodium gallinaceum ookinete adhesion to the midgut of Aedes aegypti, suggesting that carbohydrate residues are used by the parasite for recognition and binding to the midgut [7]. Furthermore, antibodies to Anopheles tessellates midgut glycoproteins that contain GalNAc side chains inhibit the infectivity of the two major human malaria parasites, P. falciparum and Plasmodium vivax [12].

Three strains of Anopheles stephensi are recorded in the south of Iran [13], but An. stephensi strain mysorensis is the dominant vector in the Sistan-Baluchistan province of southeastern Iran, where transmission of vivax malaria is particularly high. Annually about $60 \%$ of the malaria cases in Iran are reported from this province (during this study, the number of positive cases was 11,305 including 9,245 P. vivax, 1,926 P. falciparum and 134 mixed infections with both species of Plasmodium). The current annual parasite incidence (API) is 7 per 1,000 inhabitants (Center for Disease Control, Ministry of Health, Iran). In Sistan-Baluchistan province, $P$. falciparum is also resistant to anti-malaria drugs [14]. Additionally, the two most important vectors in this region, An. stephensi and Anopheles culicifacies, are both resistant to organochlorine insecticides [15]. Novel transmission-blocking and/or control strategies are urgently needed for this region of Iran. The importance of highly species- and strain-specific differences in host-parasite interactions, and consequent strategy design, cannot be overstated [16].

Therefore, the current investigation adopted a geographically-relevant study to describe the competency of the An. stephensi mysorensis vector for $P$. vivax, using insects collected from the field and parasites from patients living in the same region. The developmental kinetics of the sporogonic life cycle were investigated in detail to optimize studies on the effect of ingested carbohydrates on the two most vulnerable stages of parasite development: ooki- 
netes and sporozoites. It was found that An. stephensi mysorensis is a very competent vector for $P$. vivax, but that sporozoite invasion of the salivary glands can be powerfully blocked by the carbohydrates mannose, GalNAc, and lactose. These findings will assist the development of a specific TBV strategy targeted to this important region of Iran.

\section{Methods \\ Mosquitoes}

In order to establish a colony of wild An. stephensi strain mysorensis, mosquito larvae were collected from natural breeding paces in Iranshahr district, Sistan-Baluchistan province, Southeastern Iran, and then transferred to the insectary of a local research centre. Larvae were reared in bowls at a density of 300 larvae/500 ml of water. The pupae were transferred to cages made of muslin cloth for harbouring adults. All mosquitoes were maintained at 28 $\pm 2{ }^{\circ} \mathrm{C}$ and $70 \pm 10 \%$ relative humidity. During rearing, the adult mosquitoes were fed on $5 \%$ sucrose solution and the females on rabbit blood. The mosquitoes were allowed to regenerate three times in order to boost the population of colonies, and F3 mosquitoes were used for the experiments. For experiments (see below for details), nulliparous female mosquitoes were starved for 12-18 hours before feeding on a blood meal containing $P$. vivax gametocytes.

\section{Parasites}

Gametocytes of $P$. vivax were obtained from resident volunteer malaria patients who attended local clinics in the Iranshahr district, Baluchistan province. Patients who had gametocytes in their blood were chosen for interview and to avoid damaging the parasites, only those patients who had not previously taken any anti-malarial drugs for the current infection, were chosen as donors. Informed consent was obtained from all individuals participating in this study, and ethical approval was obtained from the Ministry of Public Health via the approvals procedure of Tehran University of Medical Sciences. All malaria patients were followed up by local Health Services personnel.

\section{Sporogonic development}

In order to determine exflagellation events, and oocyst and sporozoite development, mosquitoes were sampled at several time-points post-infection. Following infective membrane feeding, unengorged females were discarded and the fed mosquitoes were maintained in standard conditions, as described above, until assessed for sporogonic development. In order to measure the time of exflagellation, mosquitoes were dissected (five fed females each time) at one-minute intervals between one and five minutes post feeding, and then at five-minute intervals until the $30^{\text {th }}$ minute post feeding. To assess ookinete develop- ment, dissection of mosquito midguts was performed at regular intervals between 12 and 24 hours post-infection. To assess oocyst and sporozoite development, the midguts and salivary glands of mosquitoes were surveyed from 8 dpi until sporozoites were observed in the salivary glands. All dissected midguts and salivary glands were stained with mercurochrome and Giemsa (Merk Co., Germany) and observed with phase-contrast microscopy (200 and $400 \times$ magnification).

\section{Carbohydrate inhibition assay}

By characterizing P. vivax development in An. stephensi strain mysorensis, it was then possible to optimize an assay to evaluate in vivo carbohydrate inhibition effects on parasite development in the midgut and salivary glands (the two major population bottlenecks for Plasmodium). Mosquitoes were membrane-fed with gametocytaemic blood containing 0.2 M carbohydrate (based on a concentration that was optimized previously by Chen and Billingsley [17]). The following carbohydrates were selected for study because their residues had previously been detected on the surface of mosquito salivary glands and midguts $[18,19]$ : N-acetyl-glucosamine (GlcNAc), Nacetyl-galactosamine (GalNAc), arabinose, fucose, mannose, lactose, glucose and galactose. All the mosquitoes used in the sugar-feeding experiments were nulliparous females from the F3 generation cohort. For each experiment, insects were randomly separated into nine groups, with each group containing at least 100 insects. Each group was treated with a different carbohydrate (or no carbohydrate in the control group) and all nine groups were fed using the blood from a single donor. This experiment was then independently repeated a further two times, each time using 9 further groups of mosquitoes feeding on blood from a new donor. Unfed mosquitoes were discarded and the engorged mosquitoes were split into two further groups and maintained until assessed for parasite development in the midgut (at 9-10 days post-infection) or in the salivary glands (15 days post-infection), as described above.

\section{Results \\ Development of sporogony}

In this study, the development of $P$. vivax in An. stephensi strain mysorensis has been examined. Exflagellation of the parasite started within an average of one to two minutes after the conclusion of the gametocytaemic blood meal, and reached a peak of activity between 5 and 10 minutes post feeding (Figure 1). The earliest invasion of the midgut epithelium by ookinetes was observed 20 hours after blood ingestion. Mature oocysts were found between 8-14 dpi (days post infection), with the earliest oocyst rupture occurring at $9 \mathrm{~d}$ post feeding. The earliest sporozoite presence in the salivary gland was observed between day 15 and 16 post feeding (Figure 1), but estab- 


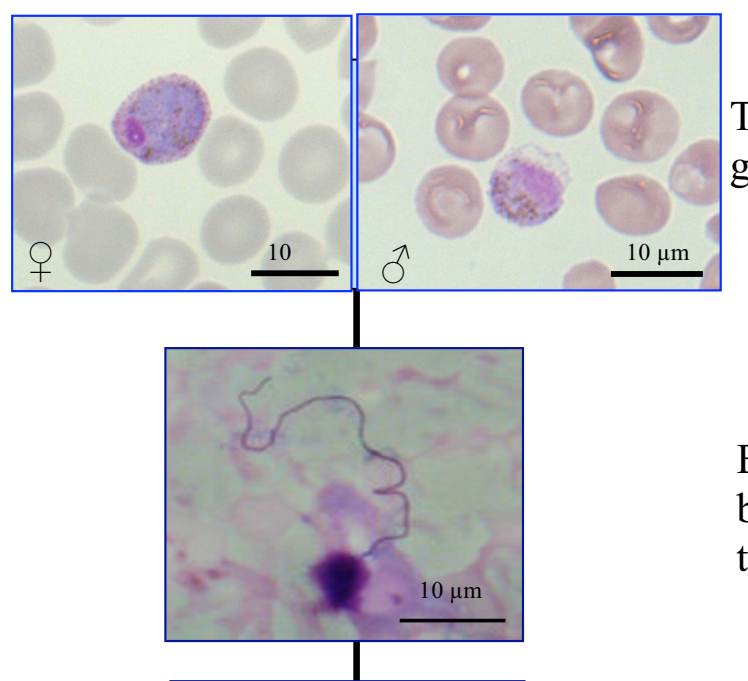

The ingested blood contains 2 to 3

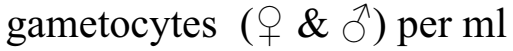

Exflagellation commences between 1 and 2 minutes after the blood meal

Ookinete development occurs in the lumen of the midgut before penetration of the epithelium at an average of 20 hours post-infection

Development of oocysts in the basal midgut occurs between 8 and 14 days post-infection
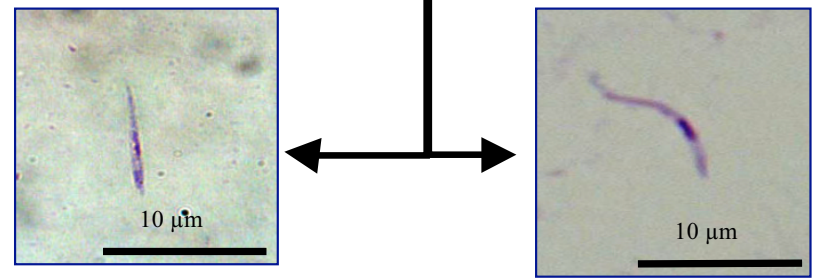

Sporozoites appear in the salivary glands on average 15 to 16 days postinfection

Sporozoites are found in haemolymph

and salivary glands simultaneously

\section{Figure I}

Sporogonic cycle and timing of the development of Plasmodium vivax in the different compartments of Anopheles stephensi strain mysorensis. Each stage of Plasmodium development was directly observed with light microscopy immediately after dissection. Giemsa staining. 
lished salivary gland infections persisted until females mosquitoes can be survived (more than 30 days). It is noteworthy that from all infected insects with $P$. vivax, the overall number of mosquitoes exhibiting sporozoite invasion of the salivary glands was $24 \%$ lower than the number supporting oocysts in their midgut $(21.9 \%$ of the infected mosquitoes exhibited live oocytes on their midguts while $16.6 \%$ of the mosquitoes' salivary glands were infected with sporozoites). Together these observations reflect the well-documented bi-phasic population bottlenecks in Plasmodium-infected mosquitoes that occur at the critical stages of ookinete invasion of the midgut, and during sporozoite invasion of the salivary glands.

\section{Carbohydrate inhibitory assays}

The effects of inhibitory carbohydrates were manifested differently at two critical stages of the P. vivax life cycle: (i) ookinete traversal of the epithelial barrier of the midgut and their subsequent development into oocysts, and (ii) sporozoites crossing into the basal membrane of salivary glands. As shown in Figure 2, inhibitor carbohydrates had a much more pronounced effect on sporozoite activities than on ookinetes/oocysts. Only minor perturbations were observed for the development of $P$. vivax oocysts at the basal side of the An. stephensi midgut following ingestion of arabinose and fucose with the gametocytemic blood meal, compared with the control group fed without carbohydrates, (Figure 2). No reductions in oocyst development were observed in mosquito groups treated with GlcNAc, GalNAc, galactose, mannose or glucose, compared with the control group.
Strikingly however, sporozoites were completely absent from salivary glands of mosquitoes treated with mannose, GalNAc, and lactose (all $\mathrm{P}<0.05$ compared with the controls; Figure 2). In contrast, sporozoite development was observed among mosquitoes treated with GlcNAc, arabinose, galactose and glucose at levels similar to those of the controls fed without carbohydrates. A small number of sporozoites were observed in the extruded haemolymph during dissections to remove the intact salivary glands. These were noted following mannose, lactose and GalNAc treatments, as well as the other carbohydrates. This suggests that the inhibitory carbohydrates acted by compromising salivary gland invasion or by intercepting haemoceol sporozoites, and not by exerting effects at earlier stages such as oocyst rupture.

\section{Discussion}

Human malaria is only transmitted via the bite of an infected mosquito, thus blocking its transmission is a potentially effective control measure. It is anticipated that this study will improve our knowledge of the role of carbohydrates in vector-parasite interactions, which can be used for developing transmission-blocking vaccines (TBVs). One important reason why a viable transmissionblocking mechanism is not yet available is because target molecules crucial to the parasite's development in the vector, are not fully understood. Furthermore, the wellknown species-specific differences in host-parasite interactions must be considered when developing TBVs targeted to specific geographical regions. From this perspective, the current two-stage study was designed to

(a) ookinetes

(b) oocysts

(c) sporozoites

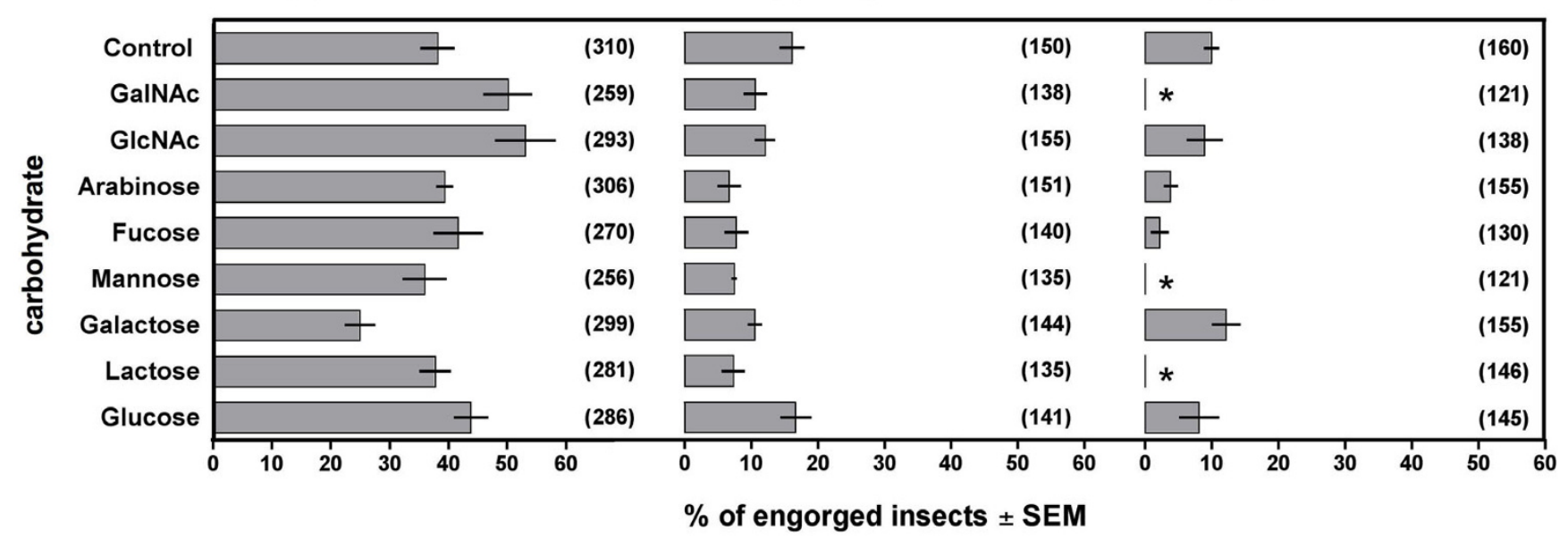

Figure 2

Inhibitory effect of carbohydrates on the development of Plasmodium vivax parasites in Anopheles stephensi mysorensis. The percentage of engorged insects exhibiting (a) ookinetes, (b) oocysts and (c) salivary gland sporozoites is reported as the mean of three independent experiments. For each of the three developmental stages, the number of insects containing parasites in each treatment group was statistically compared with the corresponding control using the Chi Squared test $(* P<0.05)$. Numbers in parentheses refer to the sample size $(n)$, i.e. total number of engorged insects. 
first examine the competency of An. stephensi strain mysorensis to support $P$. vivax development (the dominant species of Plasmodium in southern Iran), and then to identify specific carbohydrates involved in tissue localization of $P$. vivax in this mosquito host.

The results indicate that parasite development takes 12 days on average to complete its development from exflagellation to sporozoite formation. The average of adult longevity for females of An. stephensi is approximately 30 days during the malaria transmission seasons in southern Iran, and each gonotrophic cycle for this vector is $2-3$ days [20]. Thus, after 13-14 days this vector can be infectious and transfer the parasites for at least 16 days. Crucially, this means that each female has the potential to survive long enough to be re-infected and thus could transmit vivax malaria several times. A major conclusion of the study is that An. stephensi mysorensis should be considered a good vector for $P$. vivax in this particular area of Iran.

More urgent research into possible new transmission control strategies is required. This study uncovers new details about the compatibility of An. stephensi strain mysorensis with $P$. vivax, the principle vector of vivax malaria in this region of Iran. These crucial data are useful for malaria epidemiology studies in this particular region. In addition, the distribution of this mosquito strain is overlapped by another vector, An. culicifacies. It was therefore essential to clarify the role of An. stephensi strain mysorensis regarding the transmission of Plasmodium in that geographical area, which has a high incidence of malaria infections vectored by insecticide-resistant mosquitoes. It is recognized that differences in the dynamics of host-parasite interactions are strongly dependent on the mosquito/parasite species combination [21], thus, baseline information of $P$. vivax transmission in each area must be identified based on its vectors before planning effective control programs, and then control methods should address local transmission epidemiology.

The initial attachment of vector-borne pathogens to the midgut of their invertebrate hosts involves glycoprotein molecules. In the majority of arthropod vectors, the luminal face of the midgut epithelium is covered by a dense array of glycoconjugates that act as a glycan receptor for a myriad of pathogens [8]. Inhibiting this initial step with conventional methods as well as more novel approaches, such as oligosaccharide mimics or anti-glycan antibodies, could result in a reduction in disease transmission [9]. Clearly, one of the difficulties lies in developing a multivalent inhibitor that can effectively overcome the pathogen adhesion. While this is no easy task, it is likely that glycobiological analyses will make significant contributions toward resolving the mechanisms of attachment of vector-borne pathogens to their invertebrate hosts. It is known that carbohydrates taken up by a mosquito along with a parasite infective blood meal can mask parasite glycan receptors that are critical for attachment. There is evidence to suggest that malaria parasite ookinete ligands have carbohydrate recognition properties [7-22]. It can be envisaged that specific domains of the ookinete envelope glycoprotein are responsible for recognizing different glycans, or mosquito midgut microvillar glycoproteins during initial attachment. Moreover, an antibody against mosquito midgut microvillus epitopes was shown to completely inhibit Plasmodium yoelii oocyst development in the midgut of An. stephensi [10]. Furthermore, the ability of this antibody to recognize its similar mosquito carbohydrate receptor was significantly inhibited by jacalin (which is specific for Gal moieties on O-glycans) and WGA by a competition enzyme-linked immunosorbent assay [22], suggesting that the antibody shares similar glycan specificities with these two lectins.

The present study demonstrated that sporozoite invasion of the salivary glands was completely inhibited by three carbohydrates: mannose, lactose and GalNAc. Under these conditions, sporozoites were nevertheless observed in the haemolymph, strongly suggesting that the inhibitory activity of the fed carbohydrates was targeted to the salivary gland epithelium and/or the interception of sporozoites en route to the epithelium through the haemolymph. Previous studies have detected mannosyl and GlcNAc moieties on mosquito salivary glands by using FITC-labelled lectins, in three strains of An. gambiae, two strains of Anopheles arabiensis and a single strain of Anopheles merus [18]. These data collectively indicate that such sugars block their respective glycan binding proteins, which are used for sporozoite invasion.

It is postulated that sporozoite interaction with the salivary glands is species-specific [19-23] and specific glycosylated proteins might be the receptors on the basal lamina of the distal lateral and the medial lobes of the salivary glands (the regions in which sporozoites are found) [19-24]. Earlier studies also demonstrated that the invasion of $P$. gallinaceum sporozoites into the salivary glands of $A$. aegypti is blocked by a carbohydrate binding protein or lectin, wheat germ agglutinin (WGA), succinyl-WGA39 and antibody raised against this lectin [7], which confirms the role of glycosylated proteins as receptors for interaction of the sporozoite with the mosquito salivary glands. It is beyond the scope of the current study to explain why mannose, lactose and GalNAc so profoundly blocked sporozoite invasion of the salivary glands, yet did not affect midgut invasion. However, it is probable that this was due to developmental stage-specific and tissue-specific differences in host-parasite carbohydrate-receptor interactions. At present, the fields of mosquito and Plasmodium glycom- 
ics are in their infancy, but it is anticipated that future carbohydrate/oligosaccharide microarrays and other highthroughput glycan analyses will allow a detailed elucidation of stage- and tissue-specific interactions.

Therefore, vector competence is dependent on the contribution of critical glycan, and other protein co-receptors. This recognition mechanism may explain how some anopheline vectors can support mixed plasmodium infections, how some plasmodia can infect multiple anopheles vector species, or how some Anopheles are refractory to Plasmodium development.

Clearly, the relative contributions of protein or glycan receptors to Plasmodium infection of mosquitoes require additional investigation. Glycotypes are potential transmission-blocking vaccine candidates for vector-borne disease and further underline the importance of understanding the effects of carbohydrate epitopes in the development of a mosquito vaccine. TBVs typically target the development of gametocytes or ookinete interactions with the mosquito midgut. The task of blocking invasion of the salivary glands is more challenging because antibodies ingested with the blood meal must first pass through the midgut and haemocoel in a functionally intact form. However, this phenomenon is not without precedent. For example, Okulate et al [25] recently described a $73 \%$ reduction in sporozoite numbers in salivary glands of An. gambiae following ingestion of a monoclonal antibody raised against the salivary gland receptor, saglin. This antibody did not interact with the midgut and was salivary gland specific. It may therefore be possible to successfully develop TBVs based on anti-glycan antibodies, or indeed, antibodies targeting protein receptors for these carbohydrates.

The results presented here indicate that host-parasite sugar binding interactions are essential for development of $P$. vivax in the salivary glands of its vector, and the identification of the sugar binding receptors will assist further studies on TBV development.

\section{Authors' contributions}

HRB conceived, designed and coordinated the study. SD and KA conducted field studies including phlebotomy, infections and mosquito breeding. MN contributed to laboratory work including blood scrrening, and HL performed statistical analyses. HRB, SD and KA performed the experiments. HRB and MMAW wrote, read and approved the final manuscript.

\section{Acknowledgements}

The authors are indebted to the personnel of the Parasitology and Medical Entomology Department of Public Health and the Institute of Public Health Research, Tehran University of Medical Sciences and Health Training, and
Research Center of Iranshahr. The authors are also grateful to Mr. Satvat and Mrs. Motevali for laboratory assistance.

\section{References}

I. Whitten MMA, Shiao SH, Levashina EA: Mosquito midgut and malaria: cell biology, compartmentalization and immunology. Parasite Immunol 2006, 28: I 2 I - I30.

2. Billingsley PF, Baird J, Mitchell JA, Drakeley C: Immune interaction between mosquitoes and their hosts. Parasite Immunol 2006, 28: I43-I53.

3. Michel K, Kafatos FC: Mosquito immunity against Plasmodium. Insect Biochem Mol Biol 2005, 35:677-689.

4. Collins FH, Zavala F, Graves PM, Cochrane AH, Gwadz RW, Akoh J, Nussenzweig RS: First field trial of an immunoradiometric assay for the detection of malaria sporozoites in mosquitoes. Am J Trop Med Hyg 1984, 33:538-543.

5. Coleman RE, Polsa N, Eikarat N, Kollars TM Jr, Sattabongkot J: Prevention of sporogony of Plasmodium vivax in Anopheles dirus mosquitoes by transmission-blocking antimalarials. Am J Trop Med Hyg 200I, 65:214-218.

6. Osta MA, Christophides GK, Vlachou D, Kafatos FC: Innate immunity in the malaria vector Anopheles gambiae: comparative and functional genomics. J Exp Biol 2004, 207:255 I-2563.

7. Zieler H, Nawrocki JP, Shahabuddin M: Plasmodium gallinaceum ookinete adhere specifically to the midgut epithelium of Aedes aegypti by interaction with a carbohydrate ligand. Exp Parasitol 1999, 202:485-495.

8. Zieler H, Garon CF, Fischer ER, Shahabuddin M: A tubular network associated with the brush-border surface of the Aedes aegypti midgut: implications for pathogen transmission by mosquitoes. Exp Parasitol 2000, 203:I599-I6II.

9. Dinglasan RR, Jacobs-Lorena M: Insight into a conserved lifestyle: protein-carbohydrate adhesion strategies of vector-borne pathogens. Infect Immun 2005, 73:7797-7807.

10. Dinglasan RR, Fields I, Shahabuddin M, Azad AF, Sacci JB: Monoclonal antibody MG96 completely blocks Plasmodium yoelii development in Anopheles stephensi. Infect Immun 2003, 7 1:6995-7001.

II. Lal AA, Patterso PS, Sacci JB, Vaughan JA, Paul C, Collins WE, Wirtz RA, Azad AF: Anti-mosquito midgut antibodies block development of Plasmodium falciparum and Plasmodium vivax in multiple species of Anopheles mosquitoes and reduce vector fecundity and survivorship. Proc Natl Acad Sci USA 200I, 98:5228-5223.

12. Ramasamy MS, Kulasekera R, Wanniarachchi IC, Srikrishnaraj KA, Ramasamy R: Interactions of human malaria parasites, Plasmodium vivax and $P$. falciparum, with the midgut of Anopheles mosquitoes. Med Vet Entomol 1997, I I:290-296.

13. Basseri HR, Safari N, Mousakazemi SH, Akbarzadeh K: Comparison of midgut hemagglutination activity in three different geographical populations of An. stephensi. Iranian J Publ Health 2004, 33:60-67.

14. Jafari S, Le Bras J, Asmar M, Durand R: Molecular survey of Plasmodium falciparum resistance in south-eastern Iran. Ann Trop Med Parasitol 2006, 97: I 19-124.

15. Dinparast Djadid N, Barjesteh H, Raeisi A, Hassanzahi A, Zakeri S: Identification, sequence analysis and comparative study on GSTe2 insecticide resistance gene in three main world malaria vectors: Anopheles stephensi, Anopheles culicifacies, and Anopheles fluviatilis. J Med Entomol 2006, 43: I I7I-I I 77.

16. Alavi YAM, Mendoza J, Tufet-Bayona M, Sinha R, Fowler K, Billker O, Franke-Fayard B, Janse CJ, Waters A, Sinden RE: The dynamics of interactions between Plasmodium and the mosquito. Int J Parasit 2003, 33:933-943.

17. Chen C, Billingsley P: Detection and characterization of a mannan-binding lectin from mosquito, Anopheles stephensi (Liston). Eur J Biochem 1999, 263:360-366.

18. Wilkins S, Billingsley P: Partial characterization of oligosaccharides expressed on midgut microvillar glycoproteins of the mosquito, Anopheles stephensi Liston. Insect Biochem Mol Biol 2001, 3 I:937-948.

19. Mohamed HA, Ingram GA, Molyneux $\mathrm{DH}$ : Use of fluorescein labeled lectin binding of salivary glands to distinguish between Anopheles stephensi and An. albimanus species and strains. Insect Biochem 1991, 2 I:767-773. 
20. Manouchehri AV, Javadian E, Eshighy N, Motabar M: Ecology of Anopheles stephensi Liston in southern Iran. Trop Geogr Med 1976, 28:228-232.

21. Zollner GE, Ponsa N, Garman GW, Poudel S, Bell JA, Sattabongkot J, Coleman RE, Vaughan JA: Population dynamics of sporogony for Plasmodium vivax parasites from western Thailand developing within three species of colonized Anopheles mosquitoes. Malar J 2006, 5:68.

22. Dinglasan RR, Valenzuela J, Azad AF: Sugar epitopes as potential universal disease transmission blocking targets. Insect Biochem Mol Biol 2005, 35: I- 10.

23. Rosenberg R: Inability of Plasmodium knowlesi sporozoites to invade Anopheles freeborni salivary glands. Am J Trop Med Hyg 1985, 34:687-691.

24. Mohamed HA, Ingram GA: Salivary gland surface carbohydrate variations in three species of the Anopheles gambiae complex. Ann Soc Belge Med Trop 1993, 73:197-207.

25. Okulate MA, Kalume DE, Reddy R, Kristiansen T, Bhattacharyya M, Chaerkady R, Pandey A, Kumar N: Identification and molecular characterization of a novel protein Saglin as a target of monoclonal antibodies affecting salivary gland infectivity of Plasmodium sporozoites. Insect Molec Biol 2007, 16:71 I-722.

Publish with Bio Med Central and every scientist can read your work free of charge

"BioMed Central will be the most significant development for disseminating the results of biomedical research in our lifetime. "

Sir Paul Nurse, Cancer Research UK

Your research papers will be:

- available free of charge to the entire biomedical community

- peer reviewed and published immediately upon acceptance

- cited in PubMed and archived on PubMed Central

- yours - you keep the copyright

Submit your manuscript here:

http://www.biomedcentral.com/info/publishing_adv.asp 